LBNL-52580

\title{
DAMPING RING DESIGNS AND ISSUES
}

\author{
A. Wolski \\ Ernest Orlando Lawrence Berkeley National Laboratory, Berkeley, CA 94720 \\ W. Decking \\ DESY, Hamburg, Germany.
}

Presented at PAC 2003, Portland, Oregon, May 12-16, 2003

\begin{abstract}
The luminosity performance of a future linear collider (LC) will depend critically on the performance of the damping rings. The design luminosities of the current LC proposals require rings with very short damping times, large acceptance, low equilibrium emittance and high beam intensity. We discuss the design strategies for lattices achieving the goals of dynamical stability, examine the challenges for alignment and coupling correction, and consider a variety of collective effects that threaten to limit beam quality. We put the design goals in context by referring to the experience of operating facilities, and outline the further research and development that is needed.
\end{abstract}

\section{Disclaimer}

This document was prepared as an account of work sponsored by the United States Government. While this document is believed to contain correct information, neither the United States Government nor any agency thereof, nor The Regents of the University of California, nor any of their employees, makes any warranty, express or implied, or assumes any legal responsibility for the accuracy, completeness, or usefulness of any information, apparatus, product, or process disclosed, or represents that its use would not infringe privately owned rights. Reference herein to any specific commercial product, process, or service by its trade name, trademark, manufacturer, or otherwise, does not necessarily constitute or imply its endorsement, recommendation, or favoring by the United States Government or any agency thereof, or The Regents of the University of California. The views and opinions of authors expressed herein do not necessarily state or reflect those of the United States Government or any agency thereof or The Regents of the University of California.

LBNL is an equal opportunities employer.

This work was supported by the Director, Office of Science, of the U.S. Department of Energy under Contract No. DE-AC03-76SF00098. 


\title{
DAMPING RING DESIGNS AND ISSUES*
}

\author{
A. Wolski\#, LBNL, Berkeley, CA 94720, USA \\ W. Decking, DESY, Hamburg, Germany
}

\section{Abstract}

The luminosity performance of a future linear collider (LC) will depend critically on the performance of the damping rings. The design luminosities of the current LC proposals require rings with very short damping times, large acceptance, low equilibrium emittance and high beam intensity. We discuss the design strategies for lattices achieving the goals of dynamical stability, examine the challenges for alignment and coupling correction, and consider a variety of collective effects that threaten to limit beam quality. We put the design goals in context by referring to the experience of operating facilities, and outline the further research and development that is needed.

\section{PERFORMANCE SPECIFICATIONS}

The critical parameters for a linear collider damping ring are the injected and extracted emittances, repetition rate, bunch train structure, and bunch charge. Although they do not determine the damping ring design completely, these quantities place strong constraints on choices of the main design parameters, including the circumference and energy. An optimization process for a number of features of the lattice design for a linear collider damping ring has been suggested by Emma and Raubenheimer [1]. The final design is often a compromise between competing requirements. For example, a higher ring energy is favored by the need for a short damping time; however, the normalized natural emittance of a storage ring scales with the cube of the energy, so the need for a low emittance favors lower energies. The final choice of energy has to take into account collective effects, that are often more severe at low energy. There is rarely a clearcut optimum value for any free design parameter.

The different technologies adopted for the main linacs strongly affect the damping ring design, principally through the train structure. TESLA, with a superconducting linac, has a long bunch train, consisting of 2820 bunches with 337 ns bunch spacing, and a relatively low repetition rate of $5 \mathrm{~Hz}$. By contrast NLC, using a warm linac, has a train of 192 bunches with $1.4 \mathrm{~ns}$ spacing, and a higher repetition rate of $120 \mathrm{~Hz}$. To store the TESLA bunch train in a single damping ring would require a ring with a $280 \mathrm{~km}$ circumference: the present design [2] brings the ring down to a practical length by injecting and extracting individual bunches so that their spacing in the ring is only $20 \mathrm{~ns}$.

The warm machines (NLC, JLC and CLIC) have the opposite problem: the bunch trains are only approximately $80 \mathrm{~m}$ long, and to design a ring with sufficient cells in this circumference to meet the emittance specification is not

*Work supported by the US DOE under contract DE-AC03-76SF00098

\#awolski@lbl.gov practical. These machines therefore store several bunch trains at once, with each bunch trains remaining in the ring for as many machine cycles as there are trains in the ring.

The critical parameters specifying the performance of the damping rings are given in Table 1 . As usual in the context of linear colliders, the normalized emittance is quoted.

Table 1: Damping Ring Design Specifications

\begin{tabular}{|l|c|c|c|}
\hline & TESLA & NLC/JLC & CLIC \\
\hline $\mathrm{e}^{+}$emittance in $[\mu \mathrm{m}]$ & 14,000 & 45,000 & - \\
\hline $\mathrm{e}^{-}$emittance in $[\mu \mathrm{m}]$ & 40 & 150 & 10 \\
\hline Horiz. emit. out $[\mu \mathrm{m}]$ & 8 & 3 & 1.6 \\
\hline Vert. emit. out $[\mu \mathrm{m}]$ & 0.02 & 0.02 & 0.005 \\
\hline Repetition rate $[\mathrm{Hz}]$ & 5 & $120 / 150$ & 200 \\
\hline Bunches/train & 2820 & 192 & 154 \\
\hline Bunch spacing $[\mathrm{ns}]$ & 20 & 1.4 & 0.66 \\
\hline Bunch charge $\left[10^{10}\right]$ & 2 & 0.75 & 0.4 \\
\hline
\end{tabular}

The positron beam arriving at the damping rings typically has a large six-dimensional emittance. For TESLA, the positron beam is produced from an undulator source, and is compact enough that it is feasible to design a ring that has a sufficiently large acceptance and that meets the specifications for the extracted emittance. The only difference between the electron and positron damping rings for TESLA, is that the positron ring has significantly more damping wiggler. In the case of NLC/JLC, the positron beam comes from a conventional tungsten-rhenium target source, and has significantly larger transverse and longitudinal emittances. To allow for this, the NLC/JLC designs include a positron predamping ring, that has a large acceptance and is required only to damp the beam to the point that it can be accepted by a positron main damping ring that is identical to the electron main damping ring.

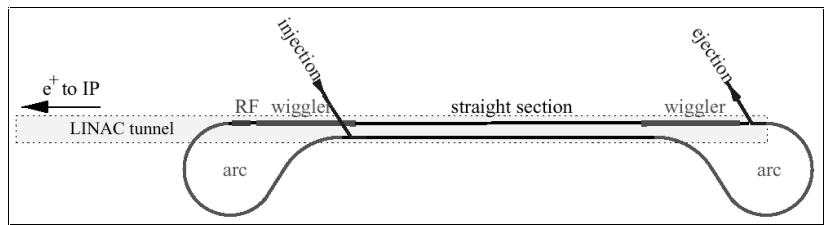

Figure 1: Conceptual Layout of the TESLA Damping Rings

The conceptual layout of one of the TESLA damping rings is shown in Figure 1. In the present design, each arc is approximately $1 \mathrm{~km}$ in circumference, and the straight sections are $7.5 \mathrm{~km}$ long and located in the main linac tunnel. The layout of the positron damping ring complex for the NLC is shown in Figure 2. The present design [3] includes a Main Damping Ring (MDR) of $300 \mathrm{~m}$ storing 3 bunch trains, and a Pre-Damping Ring (PDR) of $231 \mathrm{~m}$, 
storing 2 bunch trains. The additional circumference of the NLC rings over the lengths of the bunch trains is necessary to allow for the rise/fall times of the injection and extraction kickers. We discuss injection and extraction schemes below.

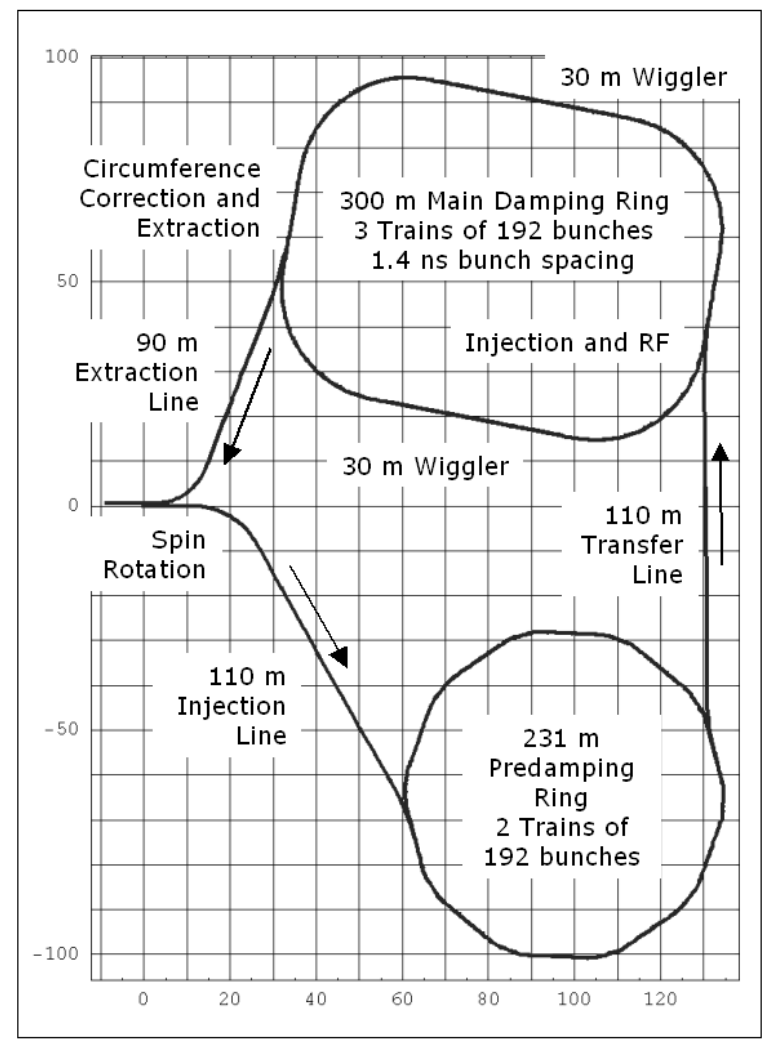

Figure 2: Layout of NLC Positron Damping Rings and Transport Lines

\section{LATTICE DESIGN}

Damping rings are similar in many respects to third generation synchrotron light sources, although the relationship is probably somewhat closer in the case of the warm machines. However, damping rings require lower natural emittances than are generally specified for light sources, and do not have the same requirement for many separate dispersion-free straight sections for insertion devices. This makes the Theoretical Minimum Emittance (TME) cell an appropriate choice for the arcs, since it allows a low natural emittance to be achieved with relatively few cells. The lattice functions in one TME arc cell in the NLC MDR are shown in Figure 3. TME cells are also used in the arcs in the CLIC and TESLA damping rings. The NLC PDR is actually a ten-fold Double Bend Achromat (DBA). A comparison between some of the parameters in the NLC MDR, the TESLA Damping Rings, the ALS, and a $5 \mathrm{GeV}$ lattice for the ESRF [4] is given in Table 2.

There are a number of considerations that need to be addressed when designing the lattice. These include:

- The natural emittance is ideally a little below the specified extracted horizontal emittance, to allow some margin for collective effects.
- With an appropriate design, the damping wigglers can significantly reduce the natural emittance.

- A large momentum compaction is needed to give a reasonably long bunch, thus reducing the charge density and reducing the impact of collective effects.

- The natural chromaticity of the lattice must be kept small, and locations provided with large dispersion and good separation of the beta functions, to minimize the chromatic sextupole strengths and give a good dynamic aperture.

- The vertical tune should be sufficiently far from an integer value, so that the closed orbit and vertical dispersion are not highly sensitive to magnet misalignments.

Table 2: Comparison of some parameters of the TESLA Positron Damping Ring, the NLC MDR, ESRF and ALS

\begin{tabular}{|l|c|c|c|c|}
\hline & TESLA & NLC & ESRF & ALS \\
\hline Circumference $[\mathrm{m}]$ & 17,000 & 300 & 845 & 197 \\
\hline Energy $[\mathrm{GeV}]$ & 5 & 1.98 & 5 & 1.9 \\
\hline Emittance $[\mu \mathrm{m}]$ & 8 & 2.4 & 25 & 25 \\
\hline Bunch length $[\mathrm{mm}]$ & 6 & 5.5 & 6 & 7 \\
\hline Energy spread $[\%]$ & 0.13 & 0.1 & 0.09 & 0.1 \\
\hline Energy loss [keV] & 21,000 & 970 & 2700 & 280 \\
\hline Damping time $[\mathrm{ms}]$ & 28 & 4.1 & 12 & 8.9 \\
\hline
\end{tabular}

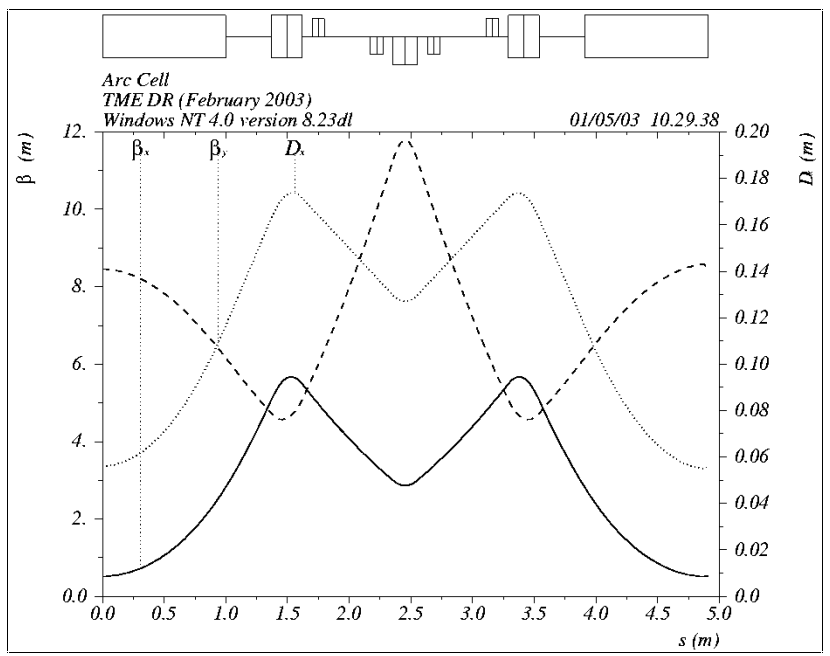

Figure 3: Lattice Functions in One TME Arc Cell in the NLC Main Damping Rings

\section{INJECTION AND EXTRACTION}

Injection schemes for synchrotron light sources generally rely on the radiation damping to merge injected off-axis bunches with stored bunches. This approach is not possible in a damping ring, since the bunch trains are stored for only a few damping times before being extracted. Instead, bunches are injected into the ring onaxis: a fast kicker is used to deflect the incoming bunches so that they have zero offset and angle with respect to the closed orbit at the exit of the kicker. Since the kicker ejects any stored charge that reaches it while it is turned on, the full bunch charge must be injected into the ring in 
a single shot. The challenges in the kicker technology are somewhat different between the different machines. The TESLA kickers must have a rise/fall time of $20 \mathrm{~ns}$, but the flat top only needs to be as long as a single bunch (some tens of picoseconds). For NLC and JLC, the rise and fall times are $65 \mathrm{~ns}$, but a flat top of around $270 \mathrm{~ns}$ is needed. Achieving the desired stability on the flat top (a fractional variation of less than $5 \times 10^{-4}$ ) can be difficult, though some success has been demonstrated with the use of double-kicker schemes to relax the tolerances [5]. The CLIC Damping Rings require kickers with a rise/fall time of $30 \mathrm{~ns}$, and a flat top of a little over $100 \mathrm{~ns}$.

\section{ACCEPTANCE}

The large average injected beam power into a damping ring (48-68 kW for the warm machines, and $226 \mathrm{~kW}$ for TESLA) means that an injection efficiency close to $100 \%$ is needed to avoid intolerable radiation loads on components in the ring. The dynamic aperture is therefore an important issue (as is the physical aperture), and is limited by the sextupoles, nonlinear field components in the damping wigglers, systematic and random multipole error components in all magnets, and tuning errors. The bare lattice (with the only significant nonlinearities coming from the sextupoles) initially needs to be carefully designed to give a large dynamic aperture. As is clear from Table 2, the damping rings include much more wiggler than is usual in a light source $(430 \mathrm{~m}$ in TESLA, $61 \mathrm{~m}$ in NLC, and $160 \mathrm{~m}$ in CLIC). The effects of the nonlinear components of the wiggler field therefore need to be carefully considered [6]. A detailed study for the NLC suggests that the wiggler effects, while visible in simulations, should not reduce the dynamic aperture to unacceptable values.

\section{VERTICAL EMITTANCE}

The specification for the extracted vertical emittance is demanding, and will require careful alignment of the magnets and rigorous coupling correction. The fundamental lower limit on the vertical emittance is placed by the opening angle of the synchrotron radiation, which excites vertical betatron oscillations even in a perfectly aligned lattice [7]. For the TESLA Damping Ring, this lower limit is approximately $1 \mathrm{~nm}$ (normalized) and for the NLC Main Damping Rings, it is approximately $0.8 \mathrm{~nm}$. In practice, much larger vertical emittance is generated both by vertical dispersion and by betatron coupling. The sensitivity of a lattice to various misalignments can be estimated from knowledge of the lattice design [8], and may be quantified by giving the rms misalignment that, on its own, will generate a specified vertical emittance. Sensitivity values for the TESLA Damping Rings, the NLC Main Damping Rings, and the ALS are compared in Table 3. A vertical emittance of $0.02 \mu \mathrm{m}$ has recently been achieved in the ALS [9]. Note that the values in Table 3 should not be interpreted as tolerances, and make no reference to skew correction of the coupling. The values quoted do, however, give an indication of the response of the vertical emittance to different misalignments for different lattices. The quadrupole jitter sensitivity quoted in Table 3 is the rms quadrupole misalignment that generates a vertical closed orbit distortion equal to the vertical beam size, which is the specified stability on the beam extracted from the damping rings. A potentially significant effect leading to emittance growth is the effect of stray fields [10].

Table 3: Sensitivity Estimates

\begin{tabular}{|l|c|c|c|}
\hline & TESLA & NLC & ALS \\
\hline Vertical emittance $[\mu \mathrm{m}]$ & 0.014 & 0.019 & 0.02 \\
\hline $\begin{array}{l}\text { Sextupole vertical } \\
\text { alignment rms }[\mu \mathrm{m}]\end{array}$ & 11 & 53 & 30 \\
\hline Quadrupole roll rms $[\mu \mathrm{rad}]$ & 38 & 511 & 200 \\
\hline Quadrupole jitter rms $[\mu \mathrm{m}]$ & 76 & 264 & 230 \\
\hline
\end{tabular}

\section{COLLECTIVE EFFECTS}

The relatively high beam intensity in the damping rings makes them vulnerable to a variety of collective instabilities that threaten to limit the operational performance. These effects range from the familiar "classical" instabilities such as those driven by the vacuum chamber impedance, to others that are less well understood, or only become significant in the new regimes in which the damping rings will operate.

\section{Microwave Instability}

The energy spread in the beam extracted from the damping rings is rotated into bunch length in the bunch compressors ahead of the main linac. It is necessary to avoid effects that increase the energy spread, that would lead directly into an increased bunch length in the linac. One such effect is the microwave instability, driven by the broad-band impedance of the vacuum chamber. Using the Boussard criterion, it is possible to estimate the threshold impedance at which the instability is expected to occur. It is usually desirable to aim for a vacuum chamber design that leaves a generous safety margin. For the TESLA DR, the impedance threshold is $\mathrm{Z} / \mathrm{n}=100 \mathrm{~m} \Omega$ for the nominal bunch charge of $2 \times 10^{10}$ particles. For the NLC MDRs, the impedance threshold is $630 \mathrm{~m} \Omega$ for the nominal bunch charge of $0.75 \times 10^{10}$. The vacuum chamber design in each case will need to be given careful consideration.

\section{Coherent Synchrotron Radiation}

The synchrotron radiation has an effect on the beam that can be described by an impedance. Heifets and Stupakov have carried out an analysis of a potential instability driven by this impedance [11], which is analogous to the microwave instability. Effects consistent with the Heifets and Stupakov theory have been observed at BESSY II and at the ALS [12]. Calculations suggested that the design of the NLC MDRs developed in 2001 would operate close to this threshold, and this motivated a redesign of the lattice to raise the threshold by increasing the momentum compaction [3]. Studies are continuing. It is possible that interference effects of the radiation in the 
wiggler can modify the form of the impedance, and this could be significant for a wiggler-dominated ring [13]. However, present indications are that in the present NLC MDR design, the threshold bunch charge for the CSR instability will be roughly an order of magnitude larger than the nominal bunch charge. The impedance scales inversely with the circumference of the ring, so for the TESLA Damping Rings, despite the long wiggler and large amount of radiation, CSR should be a small effect.

\section{Space Charge Tune Shift}

The relatively low energy of the TESLA Damping Rings for their large circumference means that there will be a large incoherent tune shift from space charge forces. A detailed analysis of the effect [14] indicated that the tune shift could result in emittance growth from particles crossing resonance lines in tune space. The proposed solution is to use combinations of skew quadrupoles to couple the beam locally in the straight sections. Tracking studies suggest that this solution would be effective in eliminating the effects of the space charge forces, and that the tolerances on tuning the "coupling bumps" are reasonable, so that it would still be possible to extract a beam with the specified vertical emittance.

\section{Intrabeam Scattering}

Intrabeam Scattering (IBS) is a familiar effect from proton machines, where the small angle scattering of particles within a bunch leads to growth of the six dimensional emittance. In electron machines, the relatively slow growth rates from IBS are usually overwhelmed by the radiation damping, but in very low emittance regimes, the bunch density can be high enough that IBS leads to an observable increase in the equilibrium emittance. Observations have been made at the KEK ATF [15] and at the ALS [16].

The IBS growth rates decrease with increasing bunch volume, and also depend strongly on the beam energy. The higher beam energy in the TESLA Damping Rings, as well as the larger beam sizes that come from the high beta functions in the long straights (apart from the coupling bumps designed to mitigate the space charge effects) mean that IBS is a comparatively small effect in the these rings. However, for the lower energy damping rings for the warm machines, IBS can potentially increase the extracted emittance above the specified values. Figure 4 shows the effect on the horizontal emittance in the NLC MDR calculated using the Bjorken-Mtingwa formalism [17]. The broken line shows the horizontal emittance decreasing from radiation damping to its equilibrium value. If IBS effects are included, as the vertical emittance approaches its equilibrium value, the IBS growth rates increase, leading to an increase in the horizontal emittance. With the nominal parameters, the extracted horizontal emittance is $3.23 \mu \mathrm{m}$, a little above the specified value of $3 \mu \mathrm{m}$.

There are a number of possible solutions that may be explored, including the use of harmonic cavities for bunch lengthening [18]. We also note that observations at the
ATF are not in full agreement with the theory, and an experimental program is continuing with the aim of resolving the discrepancy.

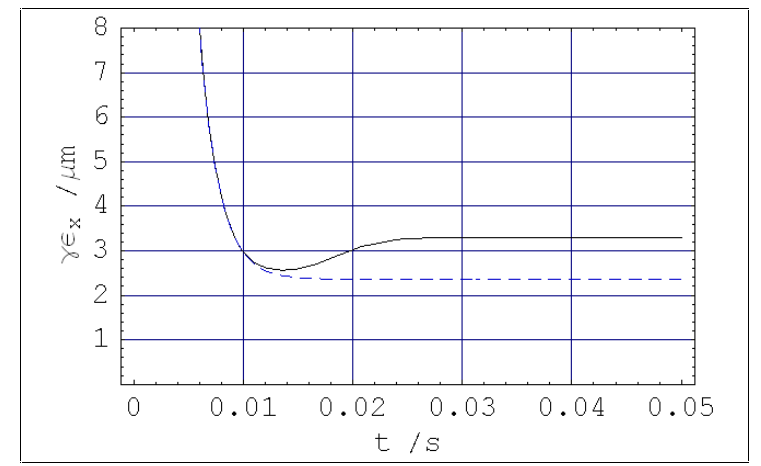

Figure 4: Effect of IBS on Horizontal Emittance in the NLC Main Damping Rings

IBS is a very strong effect in the CLIC Damping Rings, and presents significant challenges for their design [19].

\section{Electron Cloud}

Free electrons are generated in the vacuum chamber of an accelerator by a variety of processes, including photoemission, gas ionization, and secondary emission. Under certain circumstances, in positron storage rings the cloud density can reach very high levels (often saturating around the point where the electron charge in the chamber is equal to the proton or positron charge). The electron cloud can then drive single bunch or multibunch oscillations, effectively destabilizing the beam.

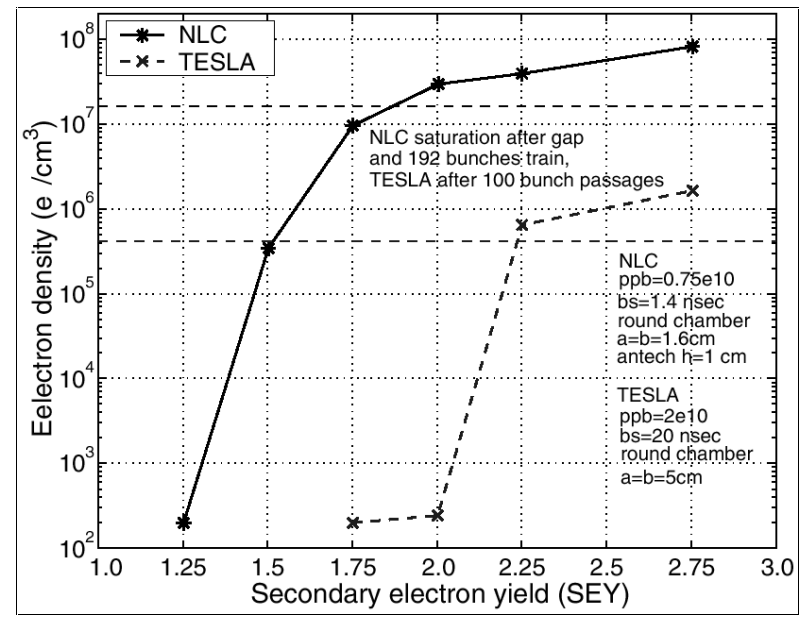

Figure 5: Mean Electron Cloud Density in the Damping Rings as a Function of Secondary Yield

Effects ascribed to electron cloud have been observed in a number of machines, notably in the low energy rings of the B Factories [20]. Estimates suggest that without taking action to prevent the build-up of the electron cloud, the impact on the operation of the TESLA and NLC/JLC Damping Rings could be severe [21]. Solenoids have provided an effective solution at the B Factories [22] by trapping secondary electrons near the vacuum chamber wall, and may be a possible solution for the TESLA Damping Rings. However, the rings for the warm 
machines are densely packed with components, and the length that may be covered by solenoids is relatively small. Instead, it is proposed to use a low secondary electron yield (SEY) coating, such as titanium nitride, to suppress the production of electrons. Figure 5 shows the mean cloud density in field-free regions of the NLC positron MDR and TESLA positron DR as a function of the peak SEY of the chamber surface [23]. It should be noted that there is still considerable uncertainty in these results, but it seems clear that the larger bunch separation in TESLA greatly reduces the build-up by allowing time for the cloud to disperse between bunches. Bare aluminum typically has a peak SEY of 2.7. Experimental studies are underway to investigate the possibility of achieving peak SEY close to 1 .

\section{Fast Ion Instability}

Ions generated during the passage of a single bunch train can couple the bunch motion, leading to increasing amplitude oscillations of bunches along the train. Although a theoretical model exists for this Fast Ion Instability [24], it has been difficult to study because of the lack of existing storage rings operating in an appropriate (very low emittance) regime. Qualitative observations have been made at the PLS, and at the ALS [25]. It is possible that the need to stay below the FII threshold will place demanding requirements on the vacuum systems, with pressures below 1 nTorr possibly needed. It is hoped that with the low coupling in the ALS achieved recently, quantitative studies may be performed to confirm the models.

\section{OUTLOOK}

Damping rings for any future linear collider will need to operate in parameter regimes not commonly achieved by existing storage rings. The designs are beginning to mature and solutions have been proposed for all the major technical challenges, although some of the suggested solutions (for example, low SEY coatings for the electron cloud) still need considerable work to be proven sufficiently effective. Experience at operating machines, for example the light sources and the KEK ATF prototype damping ring, will be crucial in developing the designs into real machines. Such experience will include optimizing the acceptance, tuning for low coupling, verifying the models for a range of collective effects, and operating the advanced diagnostics that operation of the damping rings will require.

\section{REFERENCES}

[1] P. Emma and T. Raubenheimer, "A Systematic Approach to Damping Ring Design", Phys. Rev. STAB, Volume 4, 021001, 2001.

[2] TESLA Technical Design Report, DESY 2001-011, 2001.

[3] A. Wolski et al, "A Lattice with Larger Momentum Compaction for the NLC Main Damping Rings", these proceedings.
[4] L. Hardy et al, "Recent Developments for an Improved Operation at the ESRF”, PAC'01, 2001.

[5] T. Imai et al, "Highly Stable Beam Extraction by Double Kicker System”, KEK Preprint 2002-16.

[6] M. Venturini, "Wigglers and Single Particle Dynamics in the NLC Damping Rings", these proceedings.

[7] T. Raubenheimer, "The Generation and Acceleration of Low Emittance Flat Beams for Future Linear Colliders", SLAC report 387, 1991.

[8] T. Raubenheimer and A. Wolski, "Comparison of Alignment Tolerances in Linear Collider Damping Rings with Operating Rings", Nanobeams'02.

[9] C. Steier et al, "Coupling Correction and Beam Dynamics at Ultralow Vertical Emittance in the ALS", these proceedings.

[10]W. Decking, "Influence of Klystron Stray Fields on TESLA Damping Ring", talk given at the TESLA Collaboration Meeting in Hamburg, January 2003, http://tesla.desy.de/tesla-apdg/mtgs/tesla-collab22.01.03.htm

[11] S. Heifets and G. Stupakov, "Beam Instability and Microbunching due to Coherent Synchrotron Radiation", PAC'01, p.1856.

[12] J. Byrd et al, "Observation of Broadband SelfAmplified Spontaneous Coherent Terahertz Synchrotron Radiation in a Storage Ring”, Phys. Rev. Lett. 89, 224801 (2002).

[13] J. Wu et al, "Calculation of the Coherent Synchrotron Radiation Impedance from a Wiggler", these proceedings.

[14]W. Decking and R. Brinkmann, "Space Charge Problems in the TESLA Damping Ring", EPAC'00, p.1024.

[15] K. Bane et al, "Intrabeam Scattering Analysis of ATF Beam Measurements", SLAC-PUB-8875, 2001.

[16]J. Corlett et al, "Measurements of Intrabeam Scattering at Low Emittance in the Advance Light Source", HEACC'01, 2001.

[17] J.D. Bjorken and S.K. Mtingwa, "Intrabeam Scattering", Particle Accelerators 13 p.115, 1983.

[18]S. de Santis and A. Wolski, "Harmonic Cavities for the NLC Damping Rings", these proceedings.

[19]M. Korostelev and F. Zimmermann, "A Lattice Design for the CLIC Damping Rings", DR'03, http://www.astec.ac.uk/conf/dampingring/

[20]H. Fukuma, "Electron Cloud Effects at KEK-B", Ecloud'02, CERN-2002-001. 2002.

[21]A. Wolski, "Electron Cloud in Linear Collider Damping Rings", Ecloud'02, CERN-2002-001. 2002.

[22] S.S. Win et al, "Study of Coupled Bunch Instability Caused by Electron Cloud in the KEKB Positron Ring", EPAC'02, p.1592.

[23] Thanks to Mauro Pivi (SLAC) for these results.

[24] T. Raubenheimer and F. Zimmermann, "Fast BeamIon Instability", Phys. Rev. E 52, p.5487, 1995.

[25] J. Byrd et al, "First Observations of a Fast Beam-Ion Instability at the ALS”, EPAC'98, 1998. 